\title{
Understanding and Advancing Graduate Teaching Assistants' Mathematical Knowledge for Teaching
}

\author{
Stacy Musgrave ${ }^{\mathrm{a},{ }^{*}}$, \& Marilyn P. Carlson ${ }^{\mathrm{b}}$ \\ ${ }^{a}$ Department of Mathematics and Statistics, California State Polytechnic University, Pomona, \\ 3801 W Temple Ave, Pomona CA 91768, United States, smmusgrave@cpp.edu \\ *Corresponding author. Tel: +1 909869 3135; Fax: +1 9098694616 \\ ${ }^{\mathrm{b}}$ School of Mathematical \& Statistical Sciences, Arizona State University, P.O. Box 87804, \\ Tempe AZ 85287-1804, United States, marilyn.carlson@asu.edu
}

\section{Abstract:}

Graduate student teaching assistants (GTAs) usually teach introductory level courses at the undergraduate level. Since GTAs constitute the majority of future mathematics faculty, their image of effective teaching and preparedness to lead instructional improvements will impact future directions in undergraduate mathematics curriculum and instruction. In this paper, we argue for the need to support GTAs in improving their mathematical meanings of foundational ideas and their ability to support productive student thinking. By investigating GTAs' meanings for average rate of change, a key content area in precalculus and calculus, we found evidence that even mathematically sophisticated GTAs possess impoverished meanings of this key idea. We argue for the need, and highlight one approach, for supporting GTAs to improve their understanding of foundational mathematical ideas and how these ideas are learned.

Key words: graduate student teaching assistant, mathematical meanings, average rate of change, precalculus 


\section{Understanding and Advancing Graduate Teaching Assistants' Mathematical Knowledge for Teaching}

\section{Introduction}

The results of a large national study of beginning calculus in the US revealed high attrition from calculus I to calculus II (Bressoud, Carlson, Mesa and Rasmussen, 2013). This study and others (e.g., Seymour, 2006) claim that calculus I instruction in the US frequently has a negative effect on students' mathematical confidence and their continued course-taking in mathematics. The Bressoud et al. (2013) study reported that US first semester calculus students receiving an $\mathrm{A}, \mathrm{B}$, or $\mathrm{C}$ in the course see calculus as more about learning methods for obtaining answers than understanding and using ideas to solve problems. After completing a first semester calculus course, many students also report that they are less confident in their mathematical abilities and less interested in taking more mathematics than they were upon entering the course. As universities continue to explore how to improve the mathematical success of their students, we propose that consideration for the preparation of future faculty be a central component of shifting introductory university level instruction. Graduate student teaching assistants (GTAs), namely, those individuals who constitute future faculty, teach many of the courses that undergraduate students first encounter in college (i.e., courses up to and including calculus). As such, GTAs who offer high quality instructional experiences for their students can impact a large number of students early in their studies.

As other researchers have noted, having completed many mathematics courses, as most GTAs have, does not necessarily improve a teacher's understandings and teaching practices (Speer, 2008; Speer, Gutmann, \& Murphy, 2005). Speer and Wagner (2009) further argue that the work done by mathematics instructors in providing analytic scaffolding, which entails recognizing and figuring out the ideas expressed by students to build upon and push the mathematical discussion forward, involves mathematical work. Instructors with impoverished or strictly procedural meanings for the mathematical content of a lesson will struggle to engage their students in conceptually oriented discussions around this lesson. Thompson, Carlson \& Silverman (2007) claim that:

If a teacher's conceptual structures comprise disconnected facts and procedures, their instruction is likely to focus on disconnected facts and procedures. In contrast, if a teacher's conceptual structures comprise a web of mathematical ideas and compatible ways of thinking, it will at least be possible that she attempts to develop these same conceptual structures in her students. We believe that it is mathematical understandings of the latter type that serve as a necessary condition for teachers to teach for students' highquality understanding (pp. 416-417).

The possible attempts to support student learning are revealed in the nature of the instructor's questions, her questioning patterns and the quality of the discussion she leads. All of these activities are built from how the teacher makes sense of her students' thinking, which itself is done through the lens of the teacher's own mathematical meanings (Teuscher, Moore \& Carlson, 2015).

Yet some research into the professional development of GTAs overlooks the mathematical content knowledge of the GTAs. Ellis (2014) stated:

Because graduate students come into their roles as GTAs with strong content knowledge and often little (or no) teaching experience, GTA-PD programs do not need to emphasize [...] the mathematics in the same ways that PD programs for elementary teachers specializing in mathematics may (p. 44). 
The primary aim of this report is to challenge this type of thinking about the professional development of GTAs. We report results from investigating the mathematical meanings that mathematics graduate students have for the idea of average rate of change (AROC), a key content area in precalculus and calculus. We found evidence that even mathematically sophisticated GTAs possess meanings that vary widely in productivity for teaching prior to our intervention, and that shifts in GTAs' meaning for AROC frequently require prolonged engagement in professional development focused on making meaning, building coherence and communicating with precision. In alignment with Kung \& Speer (2009), we further advocate that GTA professional development place greater emphasis on GTAs' mathematical meanings, attentiveness to student thinking, and ability to provide conceptually oriented explanations (Thompson and Thompson, 1996).

We describe the intervention we designed to improve these graduate students' meanings for this and other foundational ideas taught in a precalculus level college course. We include an expanded description of our goals for high quality instruction, how the intervention supports GTAs in making progress towards those goals, and what research has reported to be a productive meaning for the idea of AROC (Thompson, 1994). We then report on results that reveal the varied fluency among participants in speaking with meaning about AROC when probed before, during and after the intervention. We conclude with a discussion about the impact of the intervention and implications for other GTA training and professional development for undergraduate mathematics instructors.

\section{Theoretical Framework}

We provide a brief explanation of our theoretical perspective on mathematical meanings and say what we mean by high quality instruction. We also include a detailed description of the intervention used to support GTAs in developing more robust meanings of the concept of AROC.

Researchers have proposed mathematical meanings as the organization of an individual's experiences with an idea, also referred to as a scheme (Thompson, 1994). It is through repeated reasoning and reconstruction that an individual constructs schemes to organize experiences in an internally consistent way (Piaget \& Garcia, 1991; Thompson, 2013; Thompson, Carlson, Byerley, \& Hatfield, 2013). For example, an individual's meaning for the idea of AROC might consist of the calculation for the slope of a secant line, or simply $\Delta y / \Delta x$. An individual who has committed to memory that the AROC is the slope of a secant line does not possess the same meaning as someone who sees the slope of a secant line as the constant rate of change that yields the same change in the dependent quantity (as some original non-linear relationship) over the interval of the independent quantity that is of interest. These two individuals hold different meanings for the same idea, and the consequences of such differences can be profound. For instance, the former individual may or may not have an understanding of AROC as a rate of change, but instead conceptualizes AROC as the numerical output of a computation. This individual could struggle to generate problem solving strategies relying on AROC, for instance, in situations where linear approximations could help generate characterizations for how two quantities change over a given interval.

In studying individuals' meanings for various mathematical constructs, researchers are at a disadvantage - we cannot see schemes. Rather, we only have access to what an individual says, writes and gestures when engaging in mathematical activity. In our research and in the intervention, we focus on an individual's expressed meaning of an idea, or the spontaneous utterances that an individual conveys about an idea. From these utterances we can make inferences about how an individual has organized her experiences with the idea. We find the 
expressed meanings of particular interest in studying instructors' mathematical meanings because it is these spontaneous verbalizations that emerge during class instruction when students ask questions or pose solutions that deviate from what the instructor had prepared. What the instructors say in the moment, in turn, affects the ways of thinking their students develop about the mathematical idea(s) central to the discussion. We further propose that these expressed meanings provide insight into an instructor's meanings, which influence nearly all instructional activities, including: how the instructor interprets and responds to students' utterances and written products, the nature of the questions the instructor poses to students, and the selection and implementation of curricular tasks for class meetings.

We further note that an individual's meanings can be more or less productive for teaching. By more productive for teaching, we intend to describe those meanings that would support the individual in constructing new mathematical ideas and connections to other ideas, and correspondingly create the possibility for the individual to foster those connections within her students. An individual's meaning for specific ideas can be further developed through reflection, which occurs when the individual is faced with perturbations to her current meanings for those ideas (Dewey, 1910). With this perspective in mind, the intervention for GTAs is designed, in part, to perturb the GTAs' thinking about mathematical content areas specific to the courses they teach, namely precalculus and beginning calculus.

\subsection{High quality instruction}

For the purposes of this paper and the intervention being discussed, we delineate some of the characteristics we take as essential components of high quality instruction. A teacher is engaging in high quality instructional practices when she

- Supports students in constructing deep understandings and rich connections among central ideas of a course,

- Supports students in developing flexible problem solving abilities that enable them to solve problems that are novel to them and require that they apply their understandings,

- Interprets and acts on student thinking when teaching, and

- Reflects on student thinking and learning to improve teaching.

One of the behaviors that might indicate high quality instruction to an observer is that the instructor is speaking precisely about mathematical ideas, for instance, by being careful to reference quantities when describing the quantitative relationships represented in formulas and graphs (Clark, Moore, \& Carlson, 2008). Another indicator is that the instructor asks questions that elicit student thinking and then interprets, analyzes, clarifies and, whenever possible uses students' contributions to push forward the mathematical activity in the classroom (Johnson, 2013; Steffe \& Thompson, 2000). A teacher's ability to engage in these practices depends heavily on the teacher's mathematical meanings and her mathematical knowledge for teaching (Ball \& Bass, 2003; Ball, Hill, \& Bass, 2004; Silverman \& Thompson, 2008).

\subsection{A productive meaning for the idea of average rate of change}

Constructing a rich meaning of AROC entails conceptualizing a hypothetical relationship between two varying quantities in a dynamic situation. Given a relationship between the independent quantity $A$ and the dependent quantity $B$, and a fixed interval of measure of quantity $A$, the AROC of quantity $B$ with respect to quantity $A$ is the constant rate of change that yields the same change in quantity $B$ as the original relationship over the given interval. In order to understand this complex idea meaningfully, an individual must first conceptualize the idea of quantity as a measurable attribute of an object (e.g., the distance a car travels from 
home, number of minutes elapsed since noon). Note that our view of object is general and includes groups of objects (e.g., 4 people on two different relay teams) so that the objects might be two relay teams (each composed of four objects/runners) and the quantity of interest might be the distance between the two relay teams. Next, provided a situation in which two quantities vary in tandem, an individual must develop an understanding for what it means to describe the rate of change of one quantity relative to the other. Namely, the individual must conceptualize the multiplicative comparison of changes in the two quantities (the change in the output quantity is always some number of times as large as the change in the input quantity).

In the special case that the relative size of changes in one quantity relative to changes in the other remains constant, we say the quantities vary together at a constant rate of change (CROC) (see Figure 1 for the mental actions involved in constructing the idea of CROC). For example, if a car is driving at a CROC of 62.7 miles per hour, the car's change in distance will always be 62.7 times as large as an amount of (or change in) driving time (in hours).

MA 1 Recognize that quantities $A$ and $B$ covary.

MA 2 Conceptualize the change in quantity $A$ and change in quantity $B$ as quantities.

MA3 Multiplicatively compare the change in $A$ and change in $B$.

MA4 Abstract the proportional correspondence as the two quantities change together.

MA5 Conceptualize $\Delta B / \Delta A$ as the constant multiple that relates the change in quantity $A$ and the change in quantity $B$.

MA6 Connect the CROC of quantity $A$ with respect to quantity $B$ to the ratio of corresponding changes in quantity $A$ and quantity $B$ and visualize this CROC change graphically.

Figure 1. Mental actions involved in understanding constant rate of change

In the context of functional relationships, individuals with a robust meaning for AROC will see the idea of CROC as a way to characterize the overall change in a function's dependent variable for a specified interval of the independent variable. To illustrate, consider a baseball player running from first base to second base at a varying speed, completing the 90 -foot distance between the bases in 6 seconds. When watching the game, one may recognize that the player's speed initially increases and then decreases as he begins to slow down when approaching second base. A student who recognizes the relevant quantitative relationships involved in speeding up will conceptualize speeding up as running a longer distance (greater change in distance) per unit of time (some fixed change in time), and slowing down as running a shorter distance per unit of time (MA1 and MA2). The student views the idea of the CROC as a proportional relationship between the baseball player's change in distance and change in time, recognizing that if the player travels 90 feet in 6 seconds, he would travel 45 feet in 3 seconds, 15 feet in 1 second, 7.5 feet in $1 / 2$ second, and so on (MA3). The student's understanding of a proportional relationship would enable the student to conceptualize that, given any change in the amount of time run $\Delta t$, the runner's change in distance $\Delta d$ is always 15 times as large $(\Delta d=15 \Delta t)$ (MA4). To conceptualize the AROC of the runner's distance with respect to time as the player runs from first to second base involves conceptualizing and determining the CROC that would result in a 90 -foot change in distance over a 6-second time interval (MA5). The student could conceptualize that 
the CROC of the runner's distance with respect to time on this 6 second interval is represented as the slope of the secant line connecting the points $(0,0)$ and $(6,90)$ (MA6).

To further clarify what we deemed to be a desirable meaning for AROC, we include the Pathways Precalculus instructional materials (Carlson, Oehrtman \& Moore, 2015) used by the research subjects that show a general definition (Figure 2) that describes and illustrates the idea of AROC in both an algebraic and graphical context.

$<$ Figure2Pic goes here $>$

A formula of the form $y=<$ some expression that contains $x>$ describes how the values of $y$ (the dependent quantity) change in terms of varying value of $x$ (the independent quantity). The graph of the formula represents how the values of the independent and dependent quantities change together. The constant rate of change of $y$ with respect to $x$ over the interval of the independent quantity from $x_{1}$ to $x_{2}$ is \begin{tabular}{ll}
$y_{2}$ & $y_{1}$ \\
\hline$x_{2}$ & $x_{1}$
\end{tabular} . This constant rate of change is represented graphically as the slope $m$ of the straight line connecting the points $\left(x_{1}, y_{1}\right)$ and $\left(x_{2}, y_{2}\right)$.

\section{Figure 2. AROC: The CROC over an interval of the independent quantity}

Our study has been based on the premise that GTAs who hold a meaning for AROC that is strictly geometric (i.e., slope of secant line) will likely be unable to support their students in developing a quantitative meaning for AROC (Figure 2). We propose that is the quantitative meaning for AROC that an individual leverages to build connections between accumulation functions and rate of change functions, an activity foundational to applying the tools of calculus in contextualized problem scenarios. In particular, those bound by a geometric meaning for AROC may be similarly limited to thinking about derivatives in a calculus class as the result of some limiting process for slopes of secant lines. Individuals with this meaning will be hard pressed to make connections between this slope-of-tangentline meaning and, say, using the derivative to predict population values given a model of population relative to time.

\section{Subjects, the intervention and methods}

Our goal for this study was to better understand mathematics graduate students' meanings for AROC, with a particular interest in how they spontaneously describe the idea without advanced warning or coaching. To accomplish this goal, we recorded training workshops, conducted interviews and collected written data for the purpose of building characterizations of their thinking. Data collection was situated around a professional development intervention for graduate student teaching assistants at large, public universities in the United States.

\subsection{The Subjects and Data Collection}

We collected data from mathematics graduate students at three large, public, $\mathrm{PhD}$ granting universities in the United States. Participants' teaching experience varied between zero and 11 years, at both the K-12 and tertiary level. We audio-recorded an introductory workshop with 7 graduate students who were new to the intervention and curriculum to capture how novice instructors spontaneously describe the idea of AROC. We conducted semi-structured clinical interviews with 19 graduate teaching assistants, all of whom had at least one semester experience teaching a small section or acting as the recitation leader for a Precalculus course that used the Pathways Precalculus materials (Clement, 2000). The lead author conducted interviews to probe shifts in their beliefs about the roles of students and teachers in teaching and learning mathematics, and gain insights about their understandings of mathematical ideas, teaching practices and goals for student learning. Interviews were 
recorded using both a video camera and LiveScribe technology to capture audio-matched written responses to sample teaching scenarios provided during the interviews. Interviews lasted 1-2 hours, and were transcribed and coded by three members of the research team. Lastly, we collected written responses from graduate teaching assistants during the intervention to explore two curiosities: (1) Would we find greater clarity of descriptions of AROC in written form compared to oral?, and (2) Is there a distinction in types of meanings conveyed mid-intervention compared to pre- and post-intervention?

\subsection{The intervention}

The graduate students involved in the intervention volunteered to participate in the program and were compensated for their participation. All but one had met their respective university requirements to teach and agreed to further engage in a yearlong professional development program, which we call the intervention. The GTAs participated in a 2- or 3-day workshop before the start of their first semester of teaching with research-based, Pathways Precalculus curriculum materials. During this intensive workshop, the GTAs completed mathematical tasks that were designed and sequenced to support the graduate students in constructing productive meanings for the key ideas in the precalculus curriculum, including the ideas of CROC, AROC, exponential growth, angle measure, and the sine and cosine functions ${ }^{1}$. The graduate students confronted problems and questions designed to perturb their meanings for these topics. Sometimes these questions were as simple as - Eplain the meaning of ___." Other times, the workshop tasks were more advanced mathematical problems that could be solved with precalculus tools, provided the individual had a deep understanding of the mathematical ideas central to the lesson. Still other intervention tasks were situated in the act of teaching, requiring participants to respond to hypothetical student questions and responses. The intent of these questions and tasks was to prompt reflection and subsequent shifts in the GTAs' meanings for the mathematical ideas that were the focus of their instruction.

The intervention leaders, consisting of a team of individuals including at least one of the authors and members of the second author's research team (mathematics education $\mathrm{PhD}$ students involved in researching student thinking relevant to and developing curriculum for the Pathways Precalculus curriculum), conducted workshops in a manner to encourage the participants to speak with meaning about the ideas. GTAs were encouraged to avoid using vague language (e.g., use the quantity descriptions instead of pronouns, as in The distance increases" instead of ft goes up.") and to explain basic vocabulary (e.g., proportional) instead of taking terms as understood. As this type of communication was new to most of the participants, this focus on speaking with meaning continued throughout the academic year. During the fall and spring semesters, the GTAs attended weekly 90-minute seminars concurrent with teaching a course using Pathways Precalculus materials. The instructional resources use research on student thinking (e.g., Carlson, 1998; Carlson et al., 2002; Engelke, 2007; Moore, 2012; Smith, 2008; Strom, 2008) and are scaffolded to support students' construction of key ideas of precalculus that are foundational for calculus.

The primary goals of the weekly seminars were to support the graduate students in developing more productive meanings of the key ideas to be taught during the upcoming week, and to support them in clearly explaining their meanings for those ideas to others. Each of the GTAs reviewed the course materials prior to the weekly seminar and came prepared to

\footnotetext{
${ }^{1}$ The idea of average rate of change is the culminating idea of the first instructional unit of the precalculus level course the graduate students will be teaching during the upcoming semester. These ideas are highlighted because they are familiar to GTAs but difficult to discuss for novice teachers.
} 
discuss what is involved in understanding and learning the ideas central to the lesson for the subsequent week. They also gave presentations to their peers about how they intended to support their students' learning of these ideas. During the seminars, participants worked in small groups to develop mini-presentations focused on implementing the most conceptually challenging tasks to be used in the lessons for the upcoming week. At one university, GTAs were further asked to videotape their instruction and write reflections with respect to the ways of thinking they supported during that class, the types of questions they posed to students, and how they might respond differently taking student thinking into account.

The intervention had a primary focus on the mathematical content and what was entailed in understanding and learning key ideas of each lesson. GTAs and intervention leaders discussed student thinking using constructs from relevant research literature and the GTAs' classroom experiences. Materials for such discussions stemmed from the instructor materials for the Pathways Precalculus curriculum and were supplemented with both the intervention leaders' own research background and the GTAs' observations of students in their classes. Student thinking was analyzed for the purpose of describing student reasoning and understanding, and identifying productive ways for advancing students' learning. To achieve the goal of improving the GTA's ability to make sense of and advance student thinking, the intervention leaders and accompanying instructor materials had a primary focus on supporting the GTAs in developing rich and well-connected meanings for the mathematical ideas that were the focus of each lesson.

The content for the course began with a focus on developing students' ability to conceptualize quantities in a problem context and consider how pairs of varying quantities change together - these reasoning abilities have been identified to be necessary for constructing meaningful formulas and graphs (Moore \& Carlson, 2012) to define function relationships in applied contexts. As students explore the patterns of change for non-linear functions, such as exponential, quadratic, polynomial, rational, and trigonometric functions, they use the idea of AROC to characterize and compare function behavior over intervals of a function's domain. Since the idea of AROC is an important cross-cutting idea that is challenging to teach with a conceptual focus, we selected this idea for studying the GTAs' mathematical meanings. The second author's ongoing work to revise the intervention to better support GTAs in acquiring productive meanings for key ideas of precalculus suggest that focusing our study on what we had experienced to be one of (if not the) most complex idea of the course would provide greater insights into the complexities of supporting GTA's and other instructors in acquiring more robust meanings of the course's key ideas.

To further illustrate the nature of our interventions to support GTAs in providing conceptually oriented teaching, they use researcher-developed in-class investigations that include a sequence of questions scaffolded to support students in building a strong meaning for all key ideas of the course. In the case of AROC the course materials were designed to initially support students in developing foundational meanings for AROC previously described (Figures 1 and 2), with four in-class investigations containing questions that are scaffold to support students in quantitative and covariational reasoning, and to support students in understanding ideas of quantity, variable, function, proportional relationships, and CROC. The fifth investigation focuses on computing and developing the course's articulated meaning of AROC (Figure 2). The course website (used to facilitate discussions and learning during class) includes a linked applet in which two people are walking a specific distance in a fixed time, with one person walking at a CROC and the other varying her rate during the time interval being examined. The in-class investigation prompts students to explore the dynamic context by comparing the walking speed of the two individuals, examining the relative amount of time and change in distance of the two individuals as they are walking, and to 
represent the meanings they are constructing of the quantitative relationships both algebraically and graphically. This investigation includes four additional items designed to develop these same reasoning patterns. The investigation concludes by articulating and prompting students to explore the course's definition for AROC (Figure 2).

It is noteworthy that the intervention has been relatively successful in improving student learning. The quantitative data of student learning consistently shows gains from 6 to 8 points on the Precalculus Concept Assessment (PCA) instrument, a validated instrument (Carlson, Oehrtman \& Engelke, 2010) to assess students' understanding of key ideas of precalculus that have been documented to be foundational for learning calculus. Our ongoing observations of the GTAs, both when teaching and during their weekly seminars, suggest that all GTAs who participate in the intervention make noticeable shifts in their use of quantitative reasoning and their ability to support their students in constructing meaningful formulas and graphs to represent quantitative relationships in applied settings. This observation is supported by the fact that students' pre- post-scores on applied problems (e.g., Define a formula to represent the distance between a tortoise and hare as they compete in a race, given that the hare runs at a constant rate of 8 feet per second from the starting line and the tortoise, who has a head start of 22 feet, crawls at a constant rate of 1.5 feet per second.) consistently shifts from less than $20 \%$ of students constructing the correct formula to over $76 \%$ of students producing the correct formula. This is in comparison to small pre- post-shifts (19\% to $21 \%$ correct) when administered to over 1000 students enrolled in a precalculus course using a traditional curriculum, with the course's instructors receiving no professional development.

Because our observations of teachers (e.g., Tallman, 2015; Marfai, 2016) have consistently revealed that a teachers' instructional practices when teaching an idea are highly related to the teacher's meaning for and student learning of the idea, our research in the context of this project continues to investigate the complex process of supporting teachers in making significant conceptual shifts in their understanding of all key ideas of the course. With regard to the idea of AROC, our student data ${ }^{2}$ suggests that improvements in our intervention are needed, including our approach to helping teachers develop stronger meanings for this idea. The project goals include our continued adaption of the Pathways Precalculus student and instructor materials so that they become more effective in supporting its instructors to make the conceptual shifts needed to implement the Pathways Precalculus curriculum with fidelity, including each instructor's ability to articulate a coherent meaning for the course's key ideas, and to realize significant student learning gains of each key idea.

\subsection{Data Analysis}

Members of our team analyzed videos in pairs at first, identifying themes of interest relative to our conception of a productive expressed meaning for AROC before working individually to continue coding and reconvening as a group to discuss our findings (Strauss \& Corbin, 1990). We compiled our observations and identified common themes to create characterizations of verbal and written responses. After creating categories for these themes, we reviewed the data from the perspective of how we imagined a student might construct his or her meaning for AROC based on the utterance or written explanation provided by the graduate teaching assistant, should he or she produce that explanation in a classroom setting.

\section{Results}

\footnotetext{
${ }^{2}$ Only $42 \%$ of Pathways precalculus students at the end of the course provided a correct response to the PCA item that assesses student understanding of AROC.
} 
We first share data that reveals the expressed meanings that the graduate students conveyed for the idea of AROC when entering the program. We then illustrate their varied fluency in describing their meaning for AROC by sharing excerpts from clinical interviews with experienced participants. This data is then contrasted with written descriptions of participants' meaning for AROC provided the week after they had completed teaching the investigations on AROC in a precalculus course using the Pathways Precalculus materials. Collectively, our data reveals that the meanings for AROC conveyed by the novice and experienced GTAs differ in terms of the level to which they are able to spontaneously provide a meaningful description of what is involved in understanding AROC; however, it is noteworthy that even after completing the intervention, some GTAs in our study did not shift to speak fluently about the idea of AROC.

\subsection{Pre-Intervention Meanings for AROC}

As a warm-up activity for the start of a summer 2015 GTA workshop, we asked seven math graduate students to describe the meaning of AROC. Each participant's response is recorded in Figure 3, in the order in which they verbalized their meaning to the group. Their responses align with the authors' prior experiences with both students and teachers at the secondary and tertiary levels; most of the participants provided computational or geometric interpretations based on imagining a secant line between two points on the graph of a function. In particular, we see that $\mathrm{Alan}^{3}$ described AROC both computationally (i.e., $\Delta y / \Delta x$ ) and geometrically as a line, instead of highlighting the key attribute of the line - its slope.

\begin{tabular}{|rl|}
\hline Responses to the question: What does average rate of change” mean to you? \\
\hline Alan: & Delta $y$ over delta $x$. A straight line between two points on a graph \\
\hline Edgar: & $\begin{array}{l}\text { Rate of change over an interval and talk about interval as a whole. Describe the } \\
\text { rate of change. }\end{array}$ \\
\hline Cassie: & $\begin{array}{l}\text { The amount the dependent variable changes divided by the amount the } \\
\text { independent variable changes. Delta } y \text { divided by delta } x .\end{array}$ \\
\hline Diane: & Steepness of a graph [...] Uh, I don't have actual words $[\ldots]$ Slope or derivative. \\
\hline Brian: & $\begin{array}{l}\text { As one variable changes for every one unit, how much is the other variable } \\
\text { changing. Slope. Speed. }\end{array}$ \\
\hline Greg: & $\begin{array}{l}\text { I lost all the words...It's the predictive effect of changing one variable and the } \\
\text { amount and how it's going to affect the other variable. One quantity affecting } \\
\text { change in another quantity. }\end{array}$ \\
\hline
\end{tabular}

\section{Figure 3. Pre-intervention participant descriptions of AROC}

Another student, Frank, provided two equivalent descriptions of how to compute the AROC over a given interval, but did not convey what the result of those computations represent. When prompted by the workshop leader to explain the meaning of the result of the described computations, Frank struggled to communicate his thinking beyond referencing speed, saying, โThe result] represents how fast you're moving in effect. I always think of it as distance and time. It's difficult when you don't know what the quantities are." This issue of describing a meaning for AROC devoid of context arises repeatedly, even among the most experienced GTAs.

\footnotetext{
${ }^{3}$ Pseudonyms are used throughout the reporting to protect the identity of participants.
} 
Cassie and Diane spoke explicitly about a graph's steepness, a visual aspect of a graph that is simultaneously restricted to the Cartesian coordinate system and, in that setting, is potentially misleading when the coordinate axes do not have the same scale (see Figure 4). Another GTA, Brian, also mentioned slope, though he did so while conveying the idea that slope is an amount of change in the dependent quantity for each unit change in the independent quantity, a restrictive meaning for slope as it fails to support reasoning about variation when changes in the independent quantity have magnitude other than 1 . His mention of speed suggests he might have been imagining more than he communicated, but his inability, or perceived lack of need, to coherently communicate with precision about his thinking is exactly one of the characteristic behaviors of the novice GTAs the intervention aims to transform.

$<$ Figure4 goes here $>$

\section{Figure 4. Same relationship but different visual "steepness"}

Edgar's description of AROC as the rate of change over a whole interval lacks specificity and fails to communicate new information to the label of average rate of change" beyond involving an interval. Taken literally, saying the AROC describes the rate of change loses all nuances about the possibility of a situation involving a varying rate of change and how AROC allows one to capture information about a dynamic situation even in the absence of information about the actual rate of change. Greg commented on the predictive" quality of AROC, making him the only participant to explicitly highlight the idea that AROC provides an alternate means for characterizing how two quantities change together. This thinking, however, is missing many elements of what we characterize as a productive meaning for AROC.

The pre-intervention participants' expressed meanings were predominantly geometric, computational, or restricted to particular representation formats (i.e., a graphical representation of a function in the Cartesian coordinate system); moreover, only one of the seven participants spontaneously hinted at the idea that AROC serves as a tool for characterizing a function's change over some interval of its domain.

\subsection{Post-Intervention Meanings for AROC}

We analyzed 19 clinical interviews with participants who experienced at least one summer workshop and one semester of teaching with the Pathways Precalculus materials and attending the weekly seminar. In contrast to the predominantly geometric and computational descriptions of AROC from our pre-intervention participants, 15 of the 19 participants attempted to describe a meaning for AROC that conveyed some significance of the concept beyond a computation to perform (i.e., $\Delta y / \Delta x$ ) or an image to consider of a particular representation (i.e., secant line connecting two points). These descriptions can be classified as: the productive, general meaning described in our theoretical framework; a special case of that meaning for average speed; or, in one instance, a distinct interpretation the participant called tinearization." The other four participants offered explanations that fall strictly into the last four categories described in Table 1.

Table 1. Experienced participant descriptions of AROC

\begin{tabular}{|c|c|c|}
\hline $\begin{array}{c}\text { Expressed } \\
\text { Meaning } \\
\text { Category }\end{array}$ & Sample Excerpts from Clinical Interviews & $\begin{array}{c}\text { Number of } \\
\text { Instances* }\end{array}$ \\
\hline Productive - & [Students] have to understand constant rate of change & 9 \\
\hline
\end{tabular}




\begin{tabular}{|c|c|c|}
\hline General & $\begin{array}{l}\text { because the average rate of change is the constant rate of } \\
\text { change someone else would have to go, and I'm talking } \\
\text { about average speed now, to achieve the same change in } \\
\text { output for a given change in input. So, if you don't have } \\
\text { meaning for constant rate of change, well, then average rate } \\
\text { of change is just this number. }\end{array}$ & \\
\hline $\begin{array}{l}\text { Average } \\
\text { Speed }\end{array}$ & $\begin{array}{l}\text { [AROC] is a constant rate of change for that specific time } \\
\text { and distance, or uh, you know how I mean... }\end{array}$ & 8 \\
\hline $\begin{array}{l}\text { Conceptual } \\
\text { Other }\end{array}$ & $\begin{array}{l}\text { I would like to say linearization. Right, this idea of } \\
\text { approximating something that isn't linear in a linear fashion. }\end{array}$ & 1 \\
\hline Computational & $\begin{array}{l}\ldots \text { this final minus initial over the outputs and this final } \\
\text { minus initial over the inputs and that's a rate. }\end{array}$ & 4 \\
\hline Geometric & $\begin{array}{l}\text { Average rate of change is the constant rate of change to go } \\
\text { between two points. }\end{array}$ & 2 \\
\hline Incorrect & $\begin{array}{l}\text { I want my students to understand that constant rate of } \\
\text { change is a special case, I guess of average rate of change. } \\
\text { It's this special case that exists when the corresponding } \\
\text { changes in our two quantities are proportional. }\end{array}$ & 3 \\
\hline None & & 1 \\
\hline
\end{tabular}

* Total exceeds 19 because some interviewees conveyed more than one expressed meaning.

The excerpts in Table 1 suggest that the impact of the intervention on participants is far from uniform. One participant, who had four semesters of experience teaching the idea of AROC as supported by the intervention, failed to provide a clear statement of a meaning for AROC during his 14-minute response to the interview prompt, Đescribe what is involved in understanding AROC, what understandings and connections are needed to understand the idea, and how you approach teaching the idea of AROC." Of the 9 people who conveyed a productive meaning for AROC, four started with the more specific version of average speed and then generalized to a description in terms of general quantities. The other five produced explanations with various degrees of fluency, taking one participant almost 4 minutes to construct his explanation. Four of the eight participants who conveyed a meaning tied to average speed did not convey a meaning for AROC beyond comparing distance and time. In fact, two of those four GTAs only discussed average speed when prompted from the interviewer following their initial response in which they described a computation.

In analyzing the sample excerpt for a productive meaning for AROC from Table 1, we note the GTA stated AROC is the CROC satisfying certain conditions. As the GTA continued her description, noting that without a deeper understanding of CROC, AROC would simply be a number, we argue the GTA uses is" in the sense of is equal to." This usage of the verb is" was standard for GTAs conveying meanings in the top two categories of Table 1. However, not all GTAs used the same article (i.e., AROC is the versus $a$ CROC) in their descriptions. The sample excerpt for the productive general meaning of AROC shows the GTA identified AROC as the CROC satisfying certain conditions. In the sample excerpt for the average speed meaning, the GTA described AROC as $a$ CROC. The use of the indefinite article suggests average rates of change exist in a subcategory of constant rates of change, a problematic issue that the intervention might aim to address with the GTAs in the future.

The sample excerpt for an incorrect meaning suggests that the participant developed a meaning for AROC linked to CROC in a non-standard way; conventional treatment of these ideas typically describes AROC as a CROC approximation instead of viewing CROC as a special case of AROC. Another GTA stated that an AROC can be found by adding up and 
dividing, conveying a meaning for AROC that is conventionally identified as an arithmetic mean. Though not incorrect, another participant proclaimed, Iwill forever think of average rate of change as the slope of the secant line." This is not surprising since this is a common meaning conveyed in many textbooks. However, the fact that some graduate students held on to incorrect meanings, and only modest shifts were observed in others, leads us to conjecture that building a more coherent meaning for AROC is complex, especially if a less quantitative meaning (e.g., the slope of a secant line) has been previously perceived as acceptable.

Nonetheless, many participants' expressed meanings did align with our productive meaning for AROC as a way to describe a characteristic of a relationship between varying quantities, even if only in the special case of average speed. Recall that during the intervention, leaders encouraged participants to speak with meaning as a tool to support their students in reasoning about quantities. Participants were asked to use appropriate language, describe the underlying meanings of specialized vocabulary (e.g., reference quantities instead of using pronouns like it", explain proportional" instead of just using that word), and offer multiple ways of explaining a concept. We see evidence of this practice in the Productive Meaning" excerpt from Table 1 that was conveyed by a participant with 3 years of experience with the intervention, first as a participant and later as a seminar leader. Not only did she express a productive meaning for AROC, using appropriate descriptions that highlighted changes in quantities as opposed to values of quantities, she further made explicit the connection between CROC and AROC and described the mental imagery she hopes her students develop. She later elaborated the importance of students imagining a second object or scenario that displays a CROC relationship that would yield the same change in output over the given interval of the input quantity.

Similarly, a GTA with two years experience in the intervention stumbled slightly, but ultimately described AROC in terms of changes in quantities, as seen in the following interview excerpt:
I think one needs to understand that average rate of change means that [...] two quantities are varying but not necessarily at a constant rate of change- like the output quantity can, umm, not have a constant factor with respect to the input quantity. But the average rate of change of that relationship would be like if the...if there was a constant rate of change, the same output would be covered for a given amount of input. I think the easiest one for students to understand with that is the example of like distance and speed. So if you're driving your car at a constant speed and I am stopping and going and slowing down and speeding up, we will cover the same amount of distance in the same amount of time. And your - the constant rate that you go-is the same with my average rate. But I find that with that example it's really [...] hard for students to talk about things not in terms of time. I also find that using the word average" is confusing to students.

She continued to reflect on a driving context as a familiar example to support students' reasoning about AROC, but demonstrated an awareness of student thinking by highlighting that particular example as potentially problematic for students to generalize beyond contexts dependent on time. She also expressed an awareness of student difficulties with the multiple meanings of the word average" appearing in the phrase average rate of change.

Interestingly, though this GTA demonstrated a relatively high level of fluency in speaking with meaning about AROC, she pointed out that this particular idea is usually difficult for her to discuss with her students, saying:

I was struggling with it, and [...] it's just hard to word it in terms of input and output and varying quantities without having a concrete example. And so, to 
me I'm not even sure that [students are] not getting it so much as that they're not able to articulate it.

Fourteen of the 19 experienced GTAs expressed similar difficulties in discussing the idea, both during the interview and while teaching. For those GTAs who were not actively involved in the intervention and were also not the lead course instructor (they led the breakout recitation sections while a professor or lecturer provided the lecture and assigned student grades), their discomfort in expressing their meaning for AROC appeared to be more severe; not only were they unable to verbalize a coherent meaning for AROC, but they appeared more uncomfortable in being asked to do so. Examination of the video data revealed that they were more likely to squirm in their chairs, cross their arms, or move away from the interview desk. After the interviewer asked one GTA to describe his meaning for CROC, the GTA first explained how many months it had been since he taught that idea. As the interview progressed and he was asked to explain his meaning for AROC, he stumbled through saying:

The average rate of change is ...um...rather than having $a, a, u m$, ok. So the average rate of change is the, um, is again, the relation of two quantities...[explanation omitted]...ahhh...blah. You understand what I'm getting at, I hope. I'm just putting it into words poorly.

Another GTA paused for several seconds as she debated how specific to be in her response and if she would mess up writing it or verbally saying it." Both of these participants eventually conveyed a productive meaning for AROC, but not without discomfort, lengthy periods of reflection and scratch paper.

\subsection{Mid-Intervention Meanings for AROC}

Because of the difficulty some of the GTAs had in describing a meaning for AROC both before and after participating in the intervention, we gathered written data from six GTAs at one university while they were actively engaged in the intervention. Participants wrote responses to several tasks asking about their meanings for CROC and AROC during the week after they taught the idea of AROC in their respective classes, either as lead instructor or as recitation leader. We attribute some of the variation in productivity of responses to whether or not the GTA was lead instructor or recitation leader (see Table 2). All but one of the GTAs wrote a description of AROC as the CROC satisfying some condition. The first GTA produced a written description of AROC that conveys a productive meaning for AROC and uses the same level of precision in language supported in the intervention and curriculum materials. This GTA had extensive experience studying student thinking around the idea of AROC outside of her participation in the intervention and had been selected to help lead the weekly meetings of the intervention during the academic year.

Table 2. Written responses to the question "What is your meaning for 'average rate of change'?"

\begin{tabular}{|c|c|l|c|}
\hline $\begin{array}{c}\text { GTA } \\
\#\end{array}$ & $\begin{array}{c}\text { Type of } \\
\text { GTA }\end{array}$ & \multicolumn{1}{|c|}{ Written response } & $\begin{array}{c}\text { Meaning } \\
\text { Category }\end{array}$ \\
\hline 1 & $\begin{array}{c}\text { Lead } \\
\text { instructor }\end{array}$ & $\begin{array}{l}\text { I imagine } 2 \text { quantities } x \text { and } y \text { changing together, not } \\
\text { necessarily at a constant rate of change. As } x \text { changes from } \\
x_{1} \text { to } x_{2}, \text { I imagine that } y \text { changes from } y_{1} \text { to } y_{2} \cdot x_{1} \text { and } y_{1} \\
\text { stand for an arbitrary ordered pair that is fixed. The } \\
\text { average rate of change of } y \text { with respect to } x \text { over the } \\
\text { interval }\left(x_{1}, x_{2}\right) \text { is the constant rate of change needed to } \\
\left.\text { have the same change in } x \text { (that is } x_{2}-x_{1}\right) \text { and the same } \\
\text { change in } y\left(\text { that is } y_{2}-y_{1}\right) \text { as the original function. }\end{array}$ & $\begin{array}{l}\text { Productive } \\
\text { - General }\end{array}$ \\
\hline
\end{tabular}




\begin{tabular}{|c|c|c|c|}
\hline 2 & $\begin{array}{l}\text { Lead } \\
\text { instructor }\end{array}$ & $\begin{array}{l}\text { The constant rate of change needed to cover the change in } \\
\text { the output given the change in input. }\end{array}$ & \\
\hline 3 & $\begin{array}{l}\text { Recitation } \\
\text { Leader }\end{array}$ & $\begin{array}{l}\text { The constant rate of change needed to produce the same } \\
\text { change in output given the same change in input. }\end{array}$ & \\
\hline 4 & $\begin{array}{c}\text { Recitation } \\
\text { leader }\end{array}$ & $\begin{array}{l}\text { AROC is the constant rate of change required to cover the } \\
\text { same change in the dependent variable over the same } \\
\text { change in the independent variable. }\end{array}$ & \\
\hline 5 & $\begin{array}{l}\text { Recitation } \\
\text { leader }\end{array}$ & $\begin{array}{l}\text { It is the constant rate of change that you have to apply to } \\
\text { your variable to get a fixed value of your answer. For } \\
\text { example, if it is speed: it is the value of speed that you } \\
\text { should pick to get a given distance in a given amount of } \\
\text { time. }\end{array}$ & \\
\hline 6 & $\begin{array}{l}\text { Recitation } \\
\text { Leader }\end{array}$ & $\begin{array}{l}\text { It is the constant rate of change to have traveled the same } \\
\text { amount distance in the same amount of time. }\end{array}$ & $\begin{array}{l}\text { Average } \\
\text { Speed }\end{array}$ \\
\hline 7 & $\begin{array}{c}\text { Not } \\
\text { teaching }\end{array}$ & Sum all the rate of change, the divide by the total number. & $\begin{array}{l}\text { Arithmetic } \\
\text { Mean }\end{array}$ \\
\hline
\end{tabular}

In sharp contrast to the pre-intervention computational and geometric expressed meanings, GTAs 2-6 all refer to the CROC needed to achieve the -same" or given" change in one quantity relative to the -same" change in the other quantity. At this stage of the intervention, the sharpest criticism of these responses is that they neglect to clarify what these changes are the same as (i.e., a mention of an existing relationship between two quantities and the corresponding changes under inspection). In the same set of tasks, the GTAs were further prompted to construct new problem scenarios that would require a student to use the idea of AROC. All five of these GTAs produced problem scenarios that could draw upon on a productive meaning of AROC (Table 3). GTA 1 was the only GTA to construct a problem involving two non-time quantities, with her example asking a student to determine a vehicle's AROC of miles traveled with respect to gallons used based on an odometer reading and information about number of gallons purchased at a gas pump.

Table 3. Sample problem scenarios constructed by GTAs mid-intervention

\begin{tabular}{|c|c|}
\hline $\begin{array}{c}\text { GTA } \\
\#\end{array}$ & $\begin{array}{l}\text { Written responses to the prompt - Constuct a new problem scenario that would } \\
\text { require a student to use the idea of AROC to understand." }\end{array}$ \\
\hline 1 & $\begin{array}{l}\text { Andy tracks the average miles per gallon his Volvo travels. When his mpg for a } \\
\text { tank of gas is low, it suggests he might have a mechanical problem. How could } \\
\text { Andy use his odometer and the information at the gas pump to determine his } \\
\text { average rate of change of miles traveled with respect to fuel used? Does his car } \\
\text { have a constant rate of change of miles traveled with respect to gallons used? Why } \\
\text { or why not? }\end{array}$ \\
\hline 2 & $\begin{array}{l}\text { It took me } 5 \text { minutes to travel } 0.7 \text { miles from my office to class. } \\
\text { 1. If I biked at the same speed the entire way, how fast was I going? } \\
\text { 2. Was I going this fast the whole way? } \\
\text { 3. If I was rarely going this speed, why should I care about this number? Why } \\
\text { would Google Maps want this number? }\end{array}$ \\
\hline 4 & $<$ Table3Image goes here $>$ \\
\hline
\end{tabular}


GTA 7, who participated in the intervention by attending the summer workshop and the weekly seminar in hopes of getting a teaching assignment during the subsequent semester, again gave a response highlighting the stability of a meaning for AROC as the arithmetic mean by describing AROC as the arithmetic mean of rates of change. This type of response from a GTA who had completed two years of graduate courses in mathematics suggests that the weak meaning held by this student was more deep-seeded than we had initially thought. It also raises questions about the impact the intervention may have on a GTA's meanings when the GTA is not concurrently teaching with the materials.

\section{Conclusions}

The vast majority of participants held impoverished meanings for the idea of AROC at the beginning of the study, primarily focused on computation or a geometric interpretation restricted to graphical representations of function relationships in the Cartesian coordinate system. This is not a surprising result if one considers the strong procedural focus of US precalculus (Thompson and Carlson, 2017) and calculus (Tallman \& Carlson, 2012) courses, and the fact that these GTAs had not explicitly revisited these ideas in more advanced courses in mathematics. Our results further reveal that the intervention that included a summer workshop and weekly seminar while teaching precalculus using a research-based curriculum was effective in shifting these GTAs' meanings for AROC to be more conceptually focused. The majority of post-intervention interviewees attempted to give a meaning for AROC that went beyond a computational or geometric meaning for the idea. What remained variable, however, was the level of fluency of the GTAs in expressing the meaning that was an explicit goal of the intervention (Figures 1 and 2).

The initial impoverished meanings expressed by graduate students were widespread across all three institutions, suggesting that that the relatively impoverished meanings expressed by mathematics GTAs is likely a widespread phenomena and is in need of further investigation. Moreover, it bears noting that some of these graduate students had prior teaching experience, typically as recitation leader for a range of calculus classes. These findings challenge the assumptions that graduate students in mathematics have strong meanings for foundational ideas of mathematics (e.g., Ellis, 2014), and further, that having taught a course guarantees such meanings will be constructed. In fact, when asked what it means to talk about -ate of change," one GTA responded by saying, Actually, I've taught calculus before, like Calc 1. Usually in that class, I don't talk about rate of change to them. So I haven't thought about that before." Such a statement suggests that interventions such as what we have described are necessary in fostering reflective teaching practices among future faculty that might lead to more productive and coherent mathematics instruction. Failure to take action to debunk the aforementioned faulty assumptions by supporting GTAs' development of coherent and meaningful conceptions of key mathematical ideas may have severe consequences for improving the predominantly procedural focus that exists in many introductory undergraduate courses in colleges and universities across the United States of America (e.g., Tallman \& Carlson, 2012).

\section{Discussions}

Though not the focus of this research, there was mention during some of the interviews of how the Pathways Precalculus materials exposed the participants to new ways of thinking about the mathematical ideas. As one GTA said, her participation in the intervention gave her the experience of tiving conceptual learning... all of a sudden [she] was being asked questions about linear functions [she] couldn't answer and that really transformed [her] image of what precalculus was and the math that [she] was teaching." Other GTAs emphasized that 
their new ways of thinking further had positive effects on their own performance as mathematics $\mathrm{PhD}$ students. Interestingly, however, these new ways of thinking did not necessarily translate to what the participants had as goals for their students' learning. There was evidence of tension between what the GTAs had experienced as undergraduate students and what they were being asked to do as instructors with regards to discussing mathematics meaningfully.

On a similar note, experiences in working with the graduate students during the interventions produced encouraging anecdotal evidence that the opportunity to reconceptualize fundamental ideas may have a lasting impact on their image of what effective mathematics teaching entails. GTAs with prolonged exposure to the intervention (more than one year, and sometimes in conjunction with a leadership role within the intervention) more frequently commented on both wanting students to develop a more coherent view of the mathematics and the role of understanding their students' thinking in helping them become more effective instructors. One GTA even suggested that, depending on the level of influence his future position will offer, he could envision leading a similar type of program for instructors of a common course to provide a space for discussing the mathematics, student thinking and ways of supporting student learning. This leaves us optimistic that ours and other similar efforts might motivate mathematicians to engage in work to make undergraduate mathematics instruction more meaningful for students.

\section{Limitations of the Study}

A limitation of this study is that we did not include classroom data from the GTAs while teaching the idea of AROC. However, in another study reporting results of a teacher participating in the Pathways Precalculus intervention (Teuscher, Moore \& Carlson, 2015) a teacher's mathematical conception of AROC, including her knowledge of how the idea is learned (Figure 2) severely limited the teacher's ability to make sense of a student's thinking or pose questions to advance the students' understanding of AROC. To further support our conjecture, classroom observations conducted by the second author over the past 5 years, concurrent with her leading weekly seminars for GTAs participating in this research-based intervention, suggest that a teacher with weak meanings of an idea is incapable of providing conceptually coherent explanations or posing questions for the purpose of advancing a student's understanding toward the desired meaning articulated in the research-based curriculum. These observations are the primary reason that we have kept our research focus on studying the development of a GTA's meanings for the ideas she is teaching. We intend in our future research studies and call for others to investigate effective ways to support the advancement of teachers' conceptions of the mathematical ideas central to a lesson in the context of reflecting on and examining their instructional practice. We hypothesize that this more integrated approach to advancing a teacher's conceptions may lead to more immediate shifts in a teacher's instruction, including the conceptual nature of her lectures, her ability to make sense of student thinking, and to pose questions and make other instructional moves (e.g., a decision about who to call on in a whole class discussion) that engage students in ways that develop productive meanings and understanding of key concepts. We further conjecture that a teacher who is curious to understand her students' thinking and regularly reflects on her students' learning (relative to her current learning goals) will continually advance her conceptions of the ideas she is attempting to develop in her students. 


\section{Acknowledgements}

This work was supported by the National Science Foundation under Grant No. DUE1323753. Any opinions, findings and conclusions or recommendations expressed in this material are those of the author(s) and do not necessarily reflect the views of the National Science Foundation.

\section{References}

Ball, D. L., \& Bass, H. (2003). Making mathematics reasonable in school. In J. Kilpatrick, W.G. Martin, \& D. Schifter (Eds.) A Research Companion to Principles and Standards for School Mathematics (pp.27-44). Reston, VA: National Council of Teachers of Mathematics.

Ball, D. L., Hill, H.C. \& Bass, H. (2005). Knowing mathematics for teaching. Who knows mathematics well enough to teach third grade, and how can we decide? American Educator, 29(1), pp.14-17, 20-22, 43-46.

Bressoud, D.M., Carlson, M. P., Mesa, V., \& Rasmussen, C. (2013). The calculus student: insights from the Mathematical Association of America national study. International Journal of Mathematical Education in Science in Technology. 44:5, 685-698.

Carlson, M.P. (1998). A cross-sectional investigation of the development of the function concept. In A. H. Schoenfeld, J. Kaput, \& E. Dubinsky (Eds.), Research in Collegiate Mathematics Education, 3, pp.114-162. Providence, RI: American Mathematical Society.

Carlson, M.P., Jacobs, S., Coe, E., Larsen, S., \& Hsu, E. (2002) Applying Covariational Reasoning While Modeling Dynamic Events: A Framework and a Study. Journal for Research in Mathematics Education, 33(5), 352-378.

Carlson, M., Oehrtman, M., Engelke, N. (2010). The precalculus concept assessment (PCA) instrument: A tool for assessing reasoning patterns, understandings and knowledge of precalculus level students. Cognition and Instruction, 113-145.

Carlson, M.P., Oehrtman, M., \& Moore, K.C. (2015). Precalculus: Pathways to Calculus (5 ${ }^{\text {th }}$ ed.). Rational Reasoning: https://www.rationalreasoning.net

Clark, P. G., Moore, K. C., \& Carlson, M. P. (2008). Documenting the emergence of -speaking with meaning" as a sociomathematical norm in professional learning community discourse. The Journal of Mathematical Behavior, 27(4), 297-310.

Clement, J. (2000). Analysis of clinical interviews: Foundations and model viability. Handbook of research design in mathematics and science education, 547-589.

Dewey, J. (1910). How we think. Boston, D. C. Heath.

Ellis, Jessica F. (2014). Preparing future college instructors: the role of graduate student teaching assistants (GTAs) in successful college calculus programs. (Unpublished doctoral dissertation). California State University, San Diego, San Diego, CA, USA.

Engelke Infante, N.M. (2007). Students' understanding of related rates problems in calculus (Unpublished doctoral dissertation). Arizona State University, Tempe, AZ, USA.

Johnson, E. (2013). Teachers' mathematical activity in inquiry-oriented instruction. Journal of Mathematical Behavior. http://dx.doi.org/10.1016/j.jmathb.2013.03.002.

Kung, D. \& Speer, N. (2009). Teaching assistants learning to teach: Recasting early teaching experiences as rich learning opportunities. Studies in Graduate and Professional Student Development, 12, 133-152.

Marfai, F. S. (2016). Characterizing Teacher Change Through the Perturbation of Pedagogical Goals. (Unpublished doctoral dissertation). Arizona State University, Tempe, AZ, USA. 
Moore, K.C. (2012). Coherence, quantitative reasoning, and the trigonometry of students. In R. Mayes \& L.L. Hatfield (Eds.), Quantitative Reasoning and Mathematical Modeling: A Driver for STEM Integrated Education and Teaching in Context, pp.75-92. Laramie, WY: University of Wyoming.

Moore, K.C., \& Carlson, M.P. (2012). Students' images of problem contexts in solving applied problems. The Journal of Mathematical Behavior, 31(1), 48-59.

Piaget, J. and R. Garcia (1991). Toward a logic of meanings. Hillsdale, NJ, Lawrence Erlbaum.

Seymour, E. (2006). Testimony offered by Elaine Seymour, Ph.D., University of Colorado at Boulder, to the Research Subcommittee of the Committee on Science of the U.S. House of Representatives Hearing on Undergraduate Science, Math, and Engineering Education: What's Working? Wednesday, March 15, 2006.

Silverman, J. \& Thompson, P. W. (2008). Toward a framework for the development of mathematical knowledge for teaching. Journal of Mathematics Teacher Education, 11(6), 499-511.

Smith, N. (2008). Students' emergent conceptions of the fundamental theorems of calculus. (Unpublished doctoral dissertation). Arizona State University, Tempe, AZ, USA.

Speer, N. M. (2008). Connecting beliefs and practices: A fine-grained analysis of a college mathematics teacher's collections of beliefs and their relationship to his instructional practices. Cognition and Instruction, 26(2), 218-267.

Speer, N., Gutmann, T., \& Murphy, T. J. (2005). Mathematics teaching assistant preparation and development. College Teaching, 53(2), 75-80.

Speer, N.M., \& Wagner, J.F. (2009). Knowledge needed by a teacher to provide analytic scaffolding during undergraduate mathematics classroom discussions. Journal for Research in Mathematics Education, 40(5), 530-562.

Steffe, L.P., \& Thompson, P.W. (2000). Interaction or intersubjectivity? A reply to Lerman. Journal for Research in Mathematics Education, 32(2), 191-209.

Strauss, A. L., \& Corbin, J. M. (1990). Basics of qualitative research (Vol. 15). Newbury Park, CA: Sage.

Strom, A. (2008). A case study of a secondary mathematics teacher's understanding of exponential function: An emerging theoretical framework. (Unpublished doctoral dissertation). Arizona State University, Tempe, AZ, USA.

Tallman, M. A. (2015). An Examination of the Effect of a Secondary Teacher's Image of Instructional Constraints on his Enacted Subject Matter Knowledge. (Unpublished doctoral dissertation). Arizona State University, Tempe, AZ, USA.

Tallman, M. \& Carlson, M.P. (2012). A characterization of calculus I final exams in U.S. colleges and universities. Proceedings of the $15^{\text {th }}$ Annual Conference on Research in Undergraduate Mathematics Education (p. 217-226). Portland, OR: Portland State University.

Teuscher, D., Moore, K., \& Carlson, M. (2015). Decentering: A construct to analyze and explain teacher actions as they relate to student thinking. Journal of Mathematics Teacher Education, 1-24. doi: 10.1007/s10857-015-9304-0

Thompson, P. W. (2013). In the absence of meaning. In K. Leatham (Ed.), Vital directions for research in mathematics education, pp. 57-93. New York: Springer.

Thompson, P. W. (1994). The development of the concept of speed and its relationship to concepts of rate. In G. Harel \& J. Confrey (Eds.), The development of multiplicative reasoning in the learning of mathematics (pp. 179-234). Albany, NY: SUNY Press.

Thompson, P. W., Byerley, C., \& Hatfield, N. (2013). A conceptual approach to calculus made possible by technology. Computers in the Schools, 30, 124-147. 
Thompson, P. W., \& Carlson, M. P. (2017). Variation, covariation, and functions:

Foundational ways of thinking mathematically. In J. Cai (Ed.), Compendium for research in mathematics education (pp. 421-456). Reston, VA: National Council of Teachers of Mathematics.

Thompson, P. W., Carlson, M. P., \& Silverman, J. (2007). The design of tasks in support of teachers' development of coherent mathematical meanings. Journal of Mathematics Teacher Education, 10, 415-432.

Thompson, A. G., \& Thompson, P. W. (1996). Talking about rates conceptually, Part II: Mathematical knowledge for teaching. Journal for Research in Mathematics Education, 27(1), 2-24. 


\section{Figure 2}

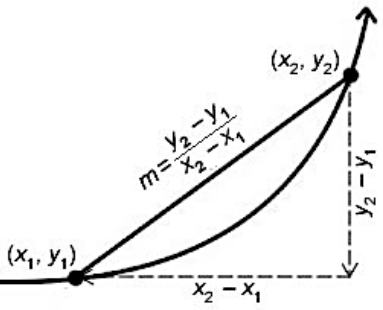

$x_{2}$ 
